\title{
Evaluation of potential circulating biomarkers for prediction of response to chemoradiation in patients with glioblastoma
}

\author{
Myra E. van Linde ${ }^{1} \cdot$ Johannes C. van der Mijn ${ }^{1} \cdot$ Thang V. Pham $^{1} \cdot$ Jaco C. Knol $^{1}$. \\ Laurine E. Wedekind $^{2} \cdot$ Koos E. Hovinga $^{3} \cdot$ Esther Sanchez Aliaga $^{4} \cdot$ Jan Buter $^{1}$. \\ Connie R. Jimenez ${ }^{1} \cdot$ Jaap C. Reijneveld $^{5} \cdot$ Henk M. W. Verheul ${ }^{1}$
}

Received: 16 December 2015 / Accepted: 4 June 2016 / Published online: 21 July 2016

(C) The Author(s) 2016. This article is published with open access at Springerlink.com

\begin{abstract}
Surgery followed by chemoradiation and adjuvant chemotherapy is standard of care for patients with a glioblastoma (GBM). Due to its limited benefit, an upfront method to predict dismal outcome would prevent unnecessary toxic treatment. We searched for a predictive blood derived biomarker in a cohort of 55 patients with GBM. Increasing age (HR 1.03, $95 \% \mathrm{CI} 1.01-1.06$ ), and postoperative tumor residue (HR 1.07, 95\% CI 1.02-1.15) were independently associated with unfavourable progression free survival (PFS) in these patients. Corticosteroid use before start of chemoradiaton was strongly predictive for outcome (HR 3.26, 95\% CI 1.67-6.39) with a mean PFS and OS in patients using corticosteroids of 7.3 and 14.6 months, versus 16.1 and 21.6 months in patients not
\end{abstract}

Myra E. van Linde and Johannes C. van der Mijn have contributed equally.

Electronic supplementary material The online version of this article (doi:10.1007/s11060-016-2178-x) contains supplementary material, which is available to authorized users.

Henk M. W. Verheul

h.verheul@vumc.nl

1 Department of Medical Oncology, VU University Medical Center, Amsterdam, The Netherlands

2 Department of Neurosurgery, Neuro-oncology Research Group, VU University Medical Center, Amsterdam, The Netherlands

3 Department of Neurosurgery, VU University Medical Center, Amsterdam, The Netherlands

4 Department of Radiology, VU University Medical Center, Amsterdam, The Netherlands

5 Department of Neurology, VU University Medical Center, Amsterdam, The Netherlands using corticosteroids $(\mathrm{p}=0.0005, \mathrm{p}<0.0067$ respectively). Despite earlier reports, blood concentrations of YKL-40, Fetuin-a and haptoglobin were not predictive for response. In addition, serum peptide profiles, determined by MALDITOF mass spectroscopy, were not predictive as well. In conclusion, further biomarker discovery studies are needed to predict treatment outcome for patients with GBM in the near future.

Keywords Glioblastoma multiforme · Biomarker . Proteomics $\cdot$ First-line treatment

\section{Introduction}

Despite intensive treatment, general prognosis of patients with a glioblastoma multiforme (GBM) remains dismal with a median survival rate of approximately 15 months and a 2 year survival rate of around $25 \%$ [1]. Current standard first-line treatment takes about 9 months to be completed and consists of surgical debulking, followed by radiotherapy with concomitant and adjuvant temozolomide [1]. This treatment regimen induces a significant burden due to its toxicity (e.g. bone marrow toxicity and fatigue), and takes a relatively long period of time. Upfront selection of GBM patients who will have no benefit from this multimodality treatment, is currently not possible. The methylation of the MGMT-gene promotor was shown to be a prognostic and a relatively weak predictive biomarker for the response to temozolomide monotherapy, and therefore clinically used only for older frail patients, when tolerability of the combination treatment is questionable [2]. IDH1 mutations were postulated as a potential predictive biomarker, but its value has to be confirmed before implementation in daily practice $[2,3]$. Assessments of both these markers and other potential genetic biomarkers require tumor tissues, which is 
not available at any given time during the treatment or follow up. Tumor tissue from patients with GBM is more difficult to obtain than a venous puncture. With a blood derived tumor marker consecutive follow-up evaluations of treatment response would be possible in order to prevent unnecessary treatment or treatment related toxicity. The availability of a blood biomarker, obtained through an easy venous puncture, predicting response to treatment in an early stage, would be an important advantage for GBM patients. It is known that proteolytic cascades within the tumor microenvironment may generate disease specific proteins and peptide fragments that are being released in blood of patients. Also proteases themselves are shed by tumors, inducing modulations of blood proteins while in the circulation as well as after blood collection ex vivo $[4,5]$. Therefore serum and plasma have been studied as a biomarker source.

In previous studies it was found that the YKL-40 concentration in serum and the YKL-40 expression in tumors is elevated $[6,7]$. Comparative genomic hybridization studies and gene expression array analysis of GBM tumor tissue revealed that YKL-40 was associated with chromosome-10 loss and poor clinical outcome [8]. Fetuin-A is one of the most abundant plasma glycoproteins and was also identified as a potential biomarker for patients with a high grade glioma. The serum FetuinA concentration, before start of treatment, was associated with survival in patients with GBM, especially when combined with age and Karnofsky performance score (KPS) [9]. Similarly, haptoglobin was found to be significantly up-regulated in serum of GBM patients and may play a role in tumor angiogenesis and proliferation of cancer cells [10]. A correlation between decreased platelet counts during concurrent radiotherapy and temozolomide treatment and a positive effect on overall survival (OS) has been described in patients with a GBM [11].

In this exploratory study, we investigated whether serum peptides as well as serum concentrations of haptoglobin, thrombocytes, YKL-40 and Fetuin-a, could predict treatment response in newly diagnosed GBM patients.

\section{Materials and methods}

\section{Patient population}

This study was conducted in the VUmc and was registered by the Medical Ethical Committee (2004/139). Each patient provided written informed consent. Included patients with histologically confirmed newly diagnosed glioblastoma received standard first-line treatment [surgery followed by irradiation (total of $60 \mathrm{~Gy})$, with concomitant temozolomide $\left(75 \mathrm{mg} / \mathrm{m}^{2}\right)$ and adjuvant six cycles of temozolomide, dosed $150-200 \mathrm{mg} / \mathrm{m}^{2}, 5$ days per week in cycles of 4 weeks]. Serum samples were collected at three different time points: postoperative, after chemoradiation, and after completion of the adjuvant treatment phase.
Postoperative tumor volume or tumor residue was calculated by using iPlan Net3.0.0 software (BrainLAB AG, Germany) by subtracting $\mathrm{T} 1$ spontaneous hyperintense signal on MRI (due to blood degradation products) in the resection cavity from the contrast enhancing residue.

Disease progression was assessed using the Macdonald criteria [12].

\section{Serum sample handling}

Venous blood was collected in $7 \mathrm{~mL}$ tubes (Cat \#367615,BD, Franklin Lakes, NJ) and allowed to clot for $30 \mathrm{~min}-1 \mathrm{~h}$ at RT. The $1 \mathrm{~h}$ at RT is an optimal time window that is needed to allow endogenous cancer specific proteases to cleave abundant blood proteins released by the clotting process, described by Villanueva et al. [5]. Blood was centrifuged at $1500 \mathrm{~g}$ for $10 \mathrm{~min}$ at RT. Serum aliquots of $300 \mu \mathrm{L}$ were taken, frozen and stored in polypropylene Eppendorf tubes at $-80^{\circ} \mathrm{C}$ until further use. All samples underwent one freeze thaw cycle before measurement. To keep pre-analytical variables as constant as possible in order to maintain the quality of the samples, a standardized, reproducible workflow has been implemented in our translational proteomic facility as described before [14].

\section{Sample spotting and MALDI-TOF mass spectrometry}

Peptide capture and measurement was performed as described before [13]. MALDI-TOF-MS was performed in reflectron positive mode on the 4800 MALDI-TOF/TOF mass spectrometer (Applied Biosystems) with 5000 shots per spectrum. The instrument was calibrated using a calibrant peptide mixture. Linear mode spectra were acquired from $m / z 800$ through 4000 .

\section{Serum protein measurements}

Protein concentrations of previously published candidate biomarkers were measured by ELISA. YKL-40 (Sunred Biological Technology Co) and Fetuin-A ( $\alpha 2$-HeremansSchmid glycoprotein, R\&D systems) measurements were performed according to manufacturer's instructions. Haptoglobin concentrations were determined by standard immunonefolometry, using a immage 800 immunochemistry system (Beckman Coulter Inc.). Thrombocyte counts were measured on a CD4000 impedance hematology analyzer.

\section{Statistical analysis}

Treatment outcome was determined by calculating time between surgery and disease progression [progression free survival (PFS) or death (OS)]. Kaplan-Meier graphs, histograms of treatment response and non-linear fitting of Gaussian distributions were performed in Graphpad prism 
(version5). Concentrations of candidate protein biomarkers were determined after log-transformation of absorbance values. Data was imported in SPSS (version20). Log transformation was applied to reach normal distributions. Pearson's correlation coefficient was determined to assess linear relationship between parameters. Serum peptide spectra were analyzed using our OPL-Analyzer software package as described before [14]. The data was pre-analyzed by preparation of metadata and the processing of raw mass spectrometry signals, which consists of peak detection, alignment, normalization and deisotoping. The peptides were further subjected to intensity filtering, requiring the median intensity of at least one group to be greater than 80 units and the fold change of the median intensities of two groups greater than 1.5 . Candidate peaks were examined visually by spectra overlay in Matlab and subjected to principle component analysis (PCA), unsupervised cluster analysis and univariate Cox regressions. All these analysis were performed without assignment of response group labels. Predictive signatures were generated by support vector machine (SVM), random forests (RF) and $k$-nearest neighbor statistical models, using peptide profiles from 24 randomly selected patients as training set. Performance of the predictive signatures was tested in the 26 remaining patients as independent test-set. Multivariate Cox regressions and subgroup analysis were performed to compare short versus long responders as determined by PFS. To view distribution of the treatment response a histogram was generated from PFS and a Gaussian distribution was fitted. The goodness of fit and $95 \%$ confidence intervals will be assessed in Graphpad prism. For the subgroup analysis the two tailed unpaired Student's $t$ test was used. All $\mathrm{p}$ values smaller than 0.05 were considered statistically significant.

\section{Results}

\section{Patient cohort and treatment response}

Between 2005 and 2012, 55 patients, were included. Table 1 summarizes the patient characteristics.

Two of the 55 patients were treated differently, with chemoradiation at a dose of $42 \mathrm{~Gy}$, without adjuvant temozolomide. The mean number of cycles completed in the adjuvant phase was five (range 1-6). In 12 patients, dose reductions with temozolomide were needed in the adjuvant phase, because of toxicity CTC grade 3 and 4 .

Follow-up of treatment response (PFS) was censored on November 8th 2012 in nine patients. Survival was censored in 18 out of 55 patients. Median PFS was 10 months (range 2-52 months) (Fig. 1a). Median OS was 15 months (range 3-52 months), and 2 year survival was $25 \%$ (Fig. $1 \mathrm{~b}$ ). Correlation between PFS as determined by the Macdonald response criteria and OS was measured. A correlation
Table 1 Patient characteristics

\begin{tabular}{|c|c|c|}
\hline Characteristic & $\begin{array}{l}\text { PFS } \\
<16 \text { months }\end{array}$ & $\begin{array}{l}\text { PFS } \\
>16 \text { months }\end{array}$ \\
\hline Patients (n) & 39 & 16 \\
\hline \multicolumn{3}{|l|}{ Age-year } \\
\hline Median & $59 *$ & $48^{*}$ \\
\hline Range & $18-75$ & $22-66$ \\
\hline \multicolumn{3}{|l|}{ Gender-no $(\%)$} \\
\hline Female & $12(31)$ & $4(25)$ \\
\hline Male & 27 (69) & $12(75)$ \\
\hline \multicolumn{3}{|l|}{ Histology-no (\%) } \\
\hline Glioblastoma & $39(100)$ & $16(100)$ \\
\hline \multicolumn{3}{|l|}{ Tumor location-no $(\%)$} \\
\hline Left & $14(36)$ & $5(31)$ \\
\hline Right & $20(51)$ & $11(69)$ \\
\hline Multifocal & $5(13)$ & - \\
\hline \multicolumn{3}{|l|}{ WHO performance } \\
\hline 0 & $22(56 \%)$ & $8(50 \%)$ \\
\hline 1 & $15(38 \%)$ & $6(38 \%)$ \\
\hline 2 & $1(3 \%)$ & \\
\hline Missing & $1(3 \%)$ & $2(13 \%)$ \\
\hline \multicolumn{3}{|l|}{ Extent of surgery } \\
\hline Biopsy & $4(10 \%)$ & - \\
\hline \multicolumn{3}{|l|}{ Debulking } \\
\hline Partial & $15(38 \%)$ & $10(63 \%)$ \\
\hline Maximal & $9(23 \%)$ & $6(38 \%)$ \\
\hline Unknown & $11(28 \%)$ & \\
\hline \multicolumn{3}{|c|}{ Time from diagnosis to radiotherapy—weeks } \\
\hline Median & 4.0 & 4.1 \\
\hline Range & $1.7-57.3$ & $3.1-5.7$ \\
\hline \multicolumn{3}{|l|}{ Radiotherapy-dose } \\
\hline $42 \mathrm{~Gy}$ & $2(5 \%)$ & \\
\hline 75 Gy & $37(95 \%)$ & $16(100 \%)$ \\
\hline \multicolumn{3}{|l|}{ TMZ-dose } \\
\hline $0 \mathrm{mg} / \mathrm{m}^{2}$ & $2(5 \%)$ & - \\
\hline $75 \mathrm{mg} / \mathrm{m}^{2}$ & $37(95 \%)$ & $16(100 \%)$ \\
\hline Dose reductions (number of pat) & $4(10 \%)$ & $2(13 \%)$ \\
\hline Average adjuvant cycles - no & 4.2 & 5 \\
\hline \multicolumn{3}{|l|}{ Corticosteroid therapy } \\
\hline Yes & $19(49 \%)^{* *}$ & $1(6 \%)^{* *}$ \\
\hline No & $20(51 \%)^{* *}$ & $15(94 \%)^{* *}$ \\
\hline \multicolumn{3}{|l|}{ Anti-epileptic therapy } \\
\hline Yes & $18(46 \%)$ & $9(56 \%)$ \\
\hline No & $20(51 \%)$ & $7(44 \%)$ \\
\hline Missing & $1(3 \%)$ & - \\
\hline
\end{tabular}

${ }^{*} \mathrm{p}=0.02$ and ${ }^{* *} \mathrm{p}=0.002$

was present with a Pearson's correlation coefficient of 0.78 $(p<0.001)$ (Fig. 1c). To view distribution of the treatment response a histogram was generated from PFS and a Gaussian distribution was fitted. The goodness of fit and $95 \%$ 
confidence intervals were assessed in Graphpad prism. The observed responses fitted well with a Gaussian distribution model with a $\mathrm{R}^{2}$ of 0.86 and narrow confidence interval for the mean (6.6-9.4 months). Mean of the Gaussian distribution was 8.0 months with a standard deviation of 4.9 months (95\% confidence interval 3.5-6.3 months) (Fig. 1d).

\section{Correlation between patient characteristics and treatment outcome}

By using univariate Cox regression analysis, significant correlations between age at the time of surgery and PFS $(p=0.008)$ between postoperative tumor residue and
PFS $(p=0.010)$, and between use of corticosteroids and PFS $(p<0.001)$ were found. No correlations were found between treatment response (PFS) and extent of resection, WHO performance score, gender, tumor location, or use of anti-epileptic drugs (Table 1). We reasoned that it would be clinically useful to identify the patients that will have long standing responses. Patients with PFS longer than 16 months ( $2 \times$ standard deviation of the mean) have substantial benefit of treatment and were therefore classified as the favorable response group and as long responders. We compared significantly associated factors in the short ( $<16$ months) versus long ( $>16$ months) responders. Both age and corticosteroids use were significantly different
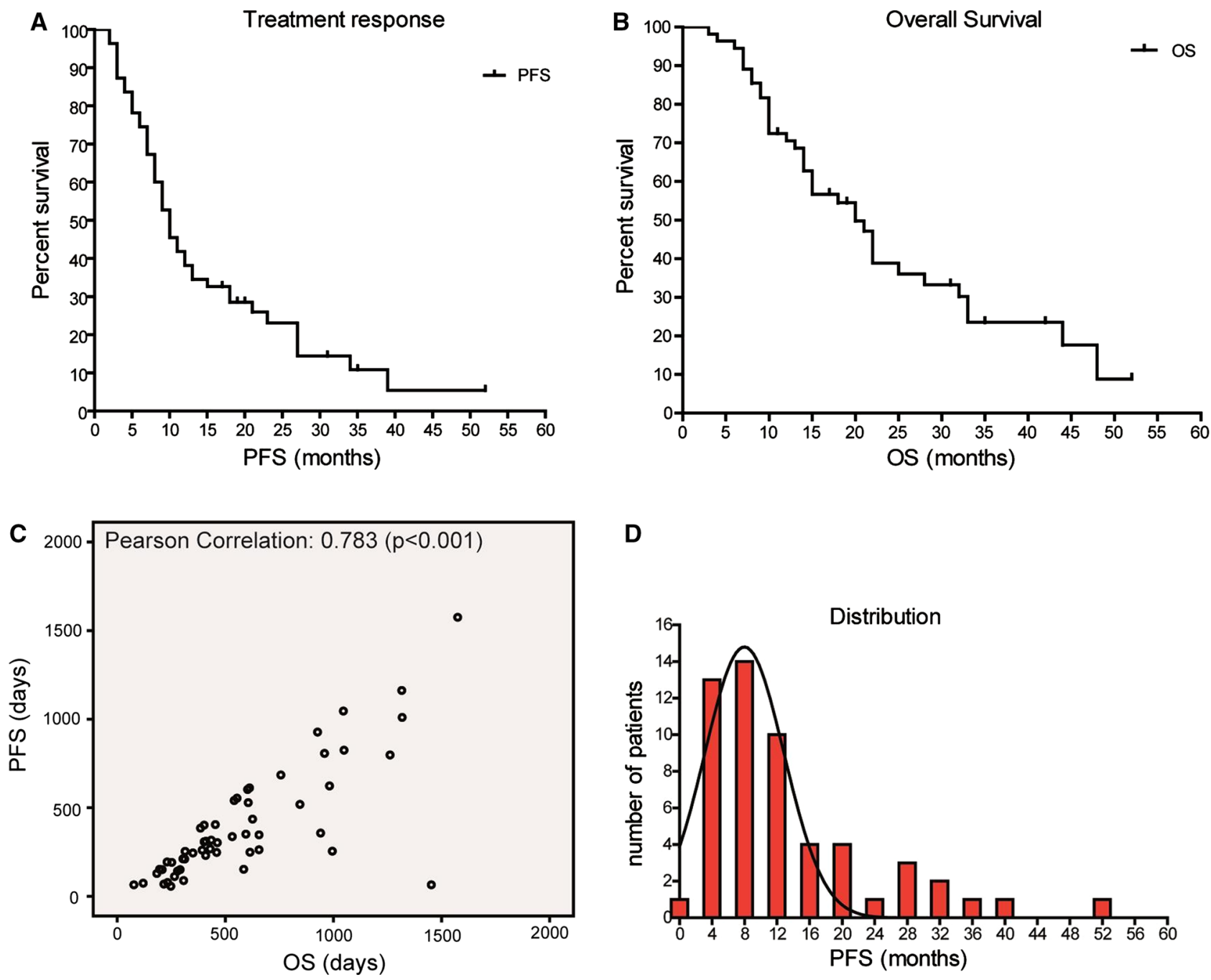

Fig. 1 a Outcome of first-line treatment in patients with glioblastoma. Treatment response of patients with glioblastoma that received radiotherapy and concomitant and adjuvant temozolomide. Kaplan-Meier curves show that (a) median PFS is 10 months (range 2-52 months). b Outcome of first-line treatment in patients with glioblastoma. Treatment response of patients with glioblastoma that received radiotherapy and concomitant and adjuvant temozolomide Kaplan-Meier curves

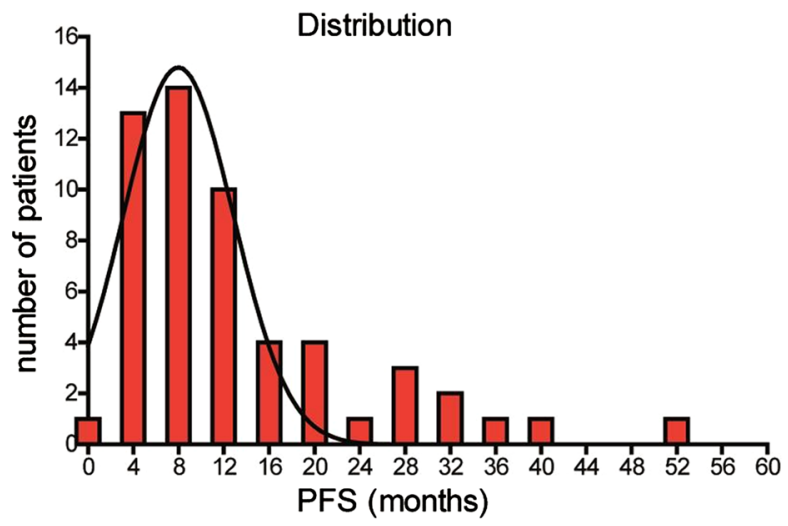

show that (b) median OS is 15 months (range 3-52 months). c Correlation between PFS and OS by Pearson correlation. Correlation between PFS and OS of patients with glioblastoma who received radiotherapy and concomitant and adjuvant temozolomide was assessed by Pearson correlation. d Normal distribution. General response (PFS) tends to follow a normal distribution with a distinct group of patients that responds beyond 16 months 
between the two response groups, while calculated postoperative tumor residue was not significantly different between the two response groups $(p=0.30)$. Multi-variate Cox regression analysis showed that both increasing age (HR 1.03, 95\% CI 1.01-1.06), postoperative tumor residue (HR 1.07, $95 \% 1.02-1.15$ ), and corticosteroid use (HR 3.26, 95\% CI 1.67-6.39) before start of chemoradiation (i.e. postoperatively) are independently associated with unfavorable treatment outcome. It is evident that only corticosteroid use before start of chemoradiation was strongly predictive for outcome. We found a significantly difference between mean PFS and OS of 7.3 and 14.6 months in patients using corticosteroids before start of chemoradiation, versus 16.1 and 21.6 months in patients not using corticosteroids before start of chemoradiation ( $p=0.0005$ and $\mathrm{p}=0.0067$, respectively) (Fig. 2a, b).

\section{No correlation between postoperative serum peptide profile and treatment response}

From 50 patients, serum was collected before start of chemoradiation. The median time between operation and the first sample taken was 21 days, the mean time was 23 days (range 13-33 days).

All serum samples were measured by MALDI-TOF mass spectrometry. After initial quality control and preprocessing of the mass spectrometry chromatograms, in total 274 peptide peaks were detected above the signal intensity threshold. Principal component analysis and unsupervised cluster analysis were performed to look at variation between samples. Both analyses showed three serum samples clustering separately from all other samples. These three serum samples were hemolytic before measurements and spectra were of poor quality and were therefore excluded from further analyses. We performed another unsupervised cluster analysis and labeled samples by gender and poor ( $<16$ months) versus favorable ( $>16$ months) treatment response (Fig. 3a). No grouping was observed by either gender or treatment response. Univariate Cox regression analysis was performed to investigate correlation between peptide levels and treatment response (PFS). In total 36 peptide peaks showed significant correlation with PFS. Visual inspection of the 36 raw peptide spectra was done and revealed 14 peaks of sufficient quality for

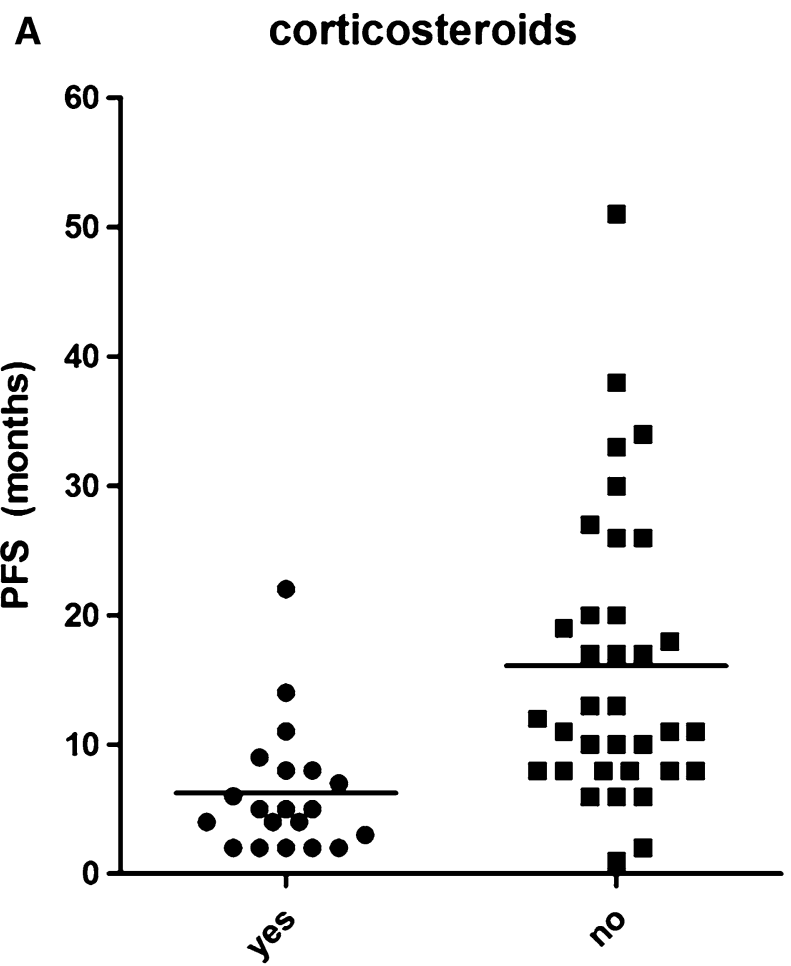

Fig. 2 a PFS in the group of patients using corticosteroids before start of chemoradiation versus the group of patients not using corticosteroids before start of chemoradiation. A significantly difference has been found between mean PFS of 7.3 months in patients using corticosteroids before start of chemoradiation versus 16.1 months in patients not using corticosteroids before start of chemoradiation $(p=0.0005)$. $\mathbf{b}$
B

\section{corticosteroids}

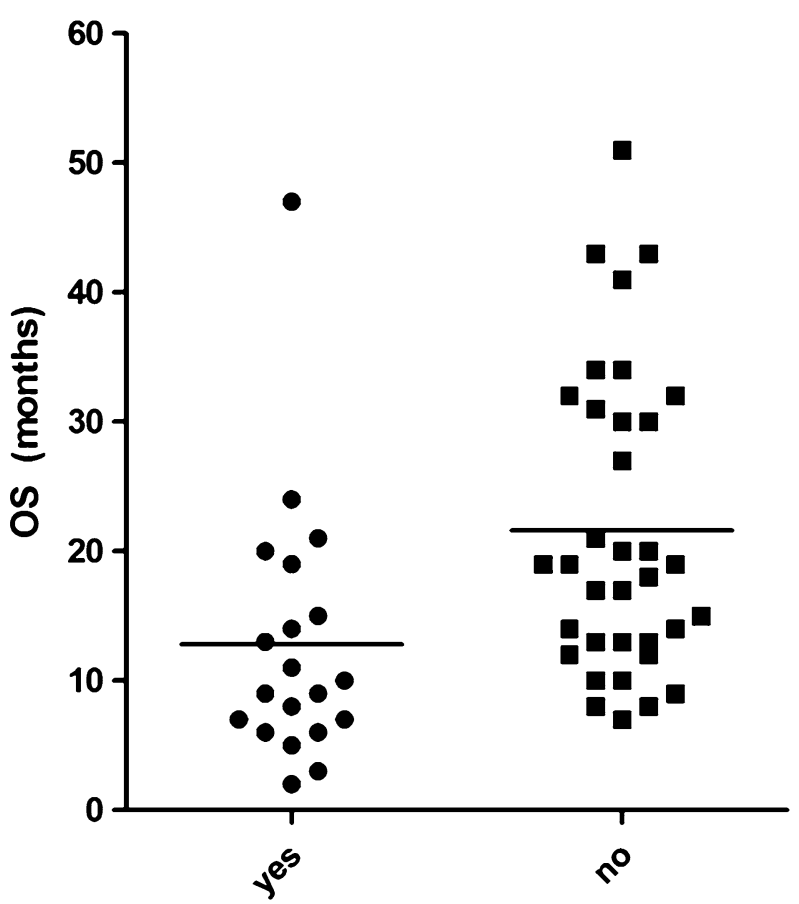

OS in the group of patients using corticosteroids before start of chemoradiation versus the group of patients not using corticosteroids before start of chemoradiation. A significantly difference has been found between mean and OS of 14.6 months in patients using corticosteroids before start of chemoradiation versus 21.6 months in patients not using corticosteroids before start of chemoradiation $(p=0.0067)$ 


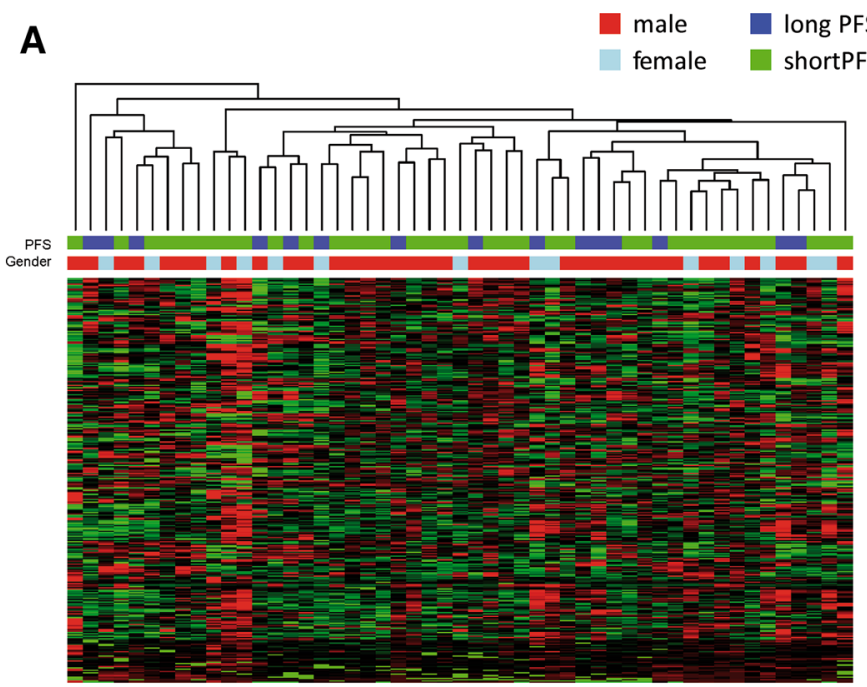

C

NM test; $p=0.23734$

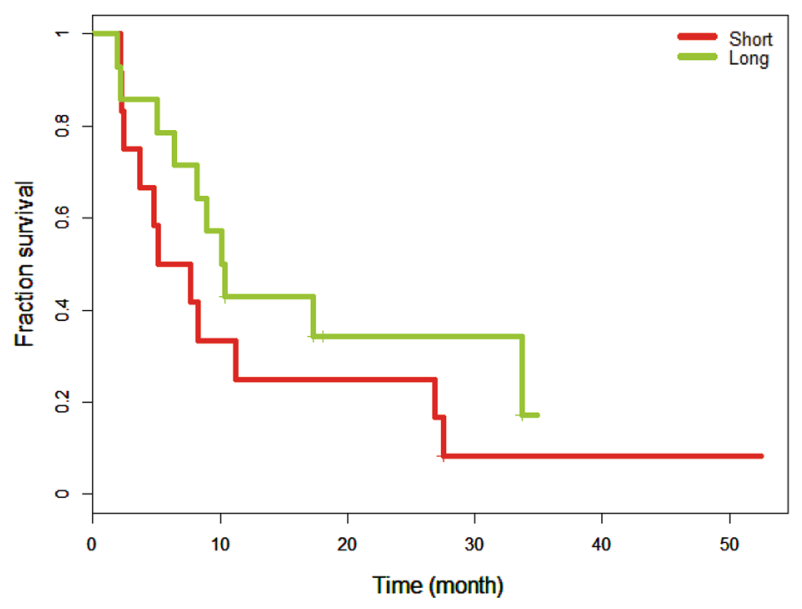

Fig. 3 a Categorization in a short versus long treatment response group based on serum peptide profiling. Serum was collected postoperatively, before start of chemoradiation and peptides were profiled by MALDI-TOF-MS. Unsupervised cluster analysis was performed and samples were labeled by gender and good (PFS $>16$ months) versus poor treatment response (PFS $<16$ months). b-d Result of testing

follow-up. After correction of p-values for multiple testing, none of the peptide peaks was significantly different between the two groups.

Despite these results in univariate analysis, we argued that combination of multiple peptides together may still yield predictive power. Therefore, we used three different statistical models to generate the best performing predictive signatures in a subset of 18 patients. Using support vector machine, a 24-peptide signature was developed for response prediction. Still, the performance of the signature in the independent testset was insufficient to separate responding from unresponsive patients $(p=0.42)$ (Fig. 3a). Similar results were obtained with different training strategies such as $k$-nearest neighbour $(p=0.24)$ and random forests $(p=0.44)$ (Fig. $3 b-d)$.
B SVM, test $26 ; p=0.41693$

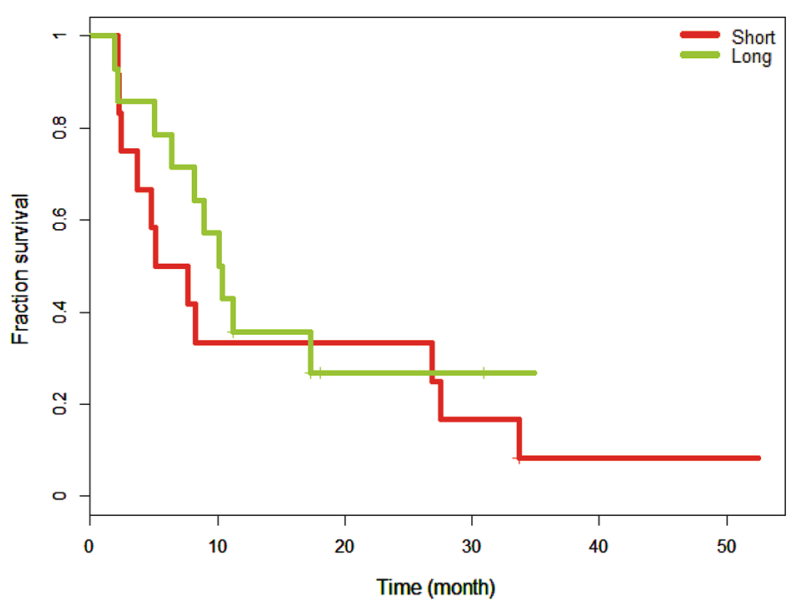

D

FR 50000 trees, test 26; $p=0.44195$

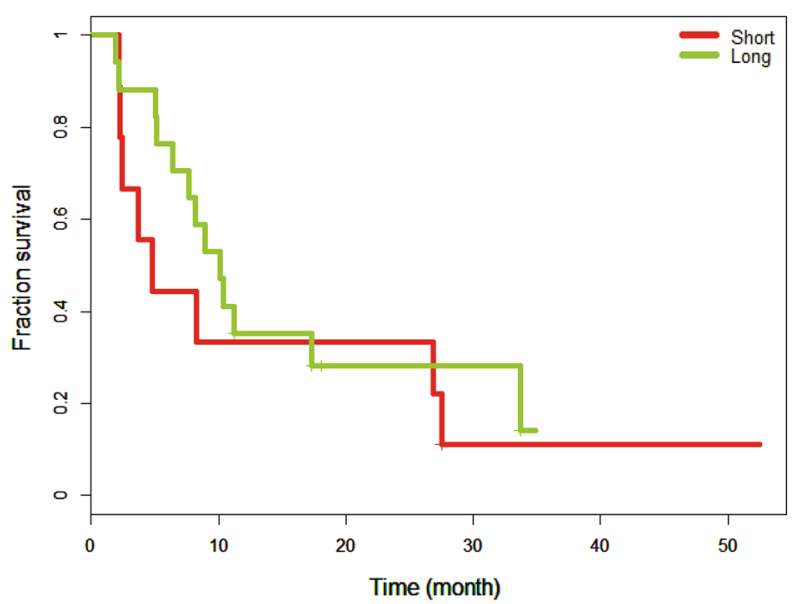

potential predictive classifiers to predict treatment response. Predictive classifiers were generated from the peptide profiles of 18 patients. Each classifier was tested in an independent patient set of 26 patients. Signatures generated by support vector machine (a), $k$-nearest neighbor (b) and random forrest (c) did not predict treatment response (b-d)

\section{Candidate protein biomarkers in serum samples}

From 47 patients, enough serum was available for measurements of haptoglobin, YKL-40, Fetuin-A and thrombocytes, after initial serum peptide profiling. Median haptoglobin concentration was $1.9 \mathrm{~g} / \mathrm{L}$ (range $0.5-4.6 \mathrm{~g} / \mathrm{L}$ ), median thrombocyte count was $288 \times 10^{9}$ cells/L (range 158 $529 \times 10^{9}$ cells/L). Haptoglobin concentration was above the reference level in 18 patients $(38 \%)$, while thrombocytosis was present in 7 patients (13\%). A significant decrease of haptoglobin concentrations was present after completing the adjuvant phase, but not after completing chemoradiation ( $p<0.0001$, Suppl.Fig.S1). No significant change in thrombocyte counts was found (Suppl.Fig.S2). Similarly, 
no significant correlation was found between haptoglobin concentrations $(p=0.16)$ or thrombocyte counts and PFS. Median YKL-40 and Fetuin-A concentrations were $37.8 \mathrm{ng} /$ $\mathrm{mL}$ (range 21.8-183.3 ng/mL) and $460.9 \mathrm{mg} / \mathrm{L}$ (range 236.4-901.4 mg/L) respectively. Both YKL-40 and FetuinA concentrations were significantly increased, when comparing the pre-chemoradiation samples with the samples after chemoradiation ( $\mathrm{p}=0.0002, \mathrm{p}=0.0026$, suppl.Fig.S3, S4). Both YKL-40 and Fetuin-A concentrations were not associated with PFS as determined by Cox regression analysis ( $p=0.54$ and $p=0.25$ respectively). However, when comparing concentrations in our previously identified response groups, Fetuin-A was significantly increased in patients with favorable treatment response $(\mathrm{p}=0.03)$ (Fig. 4a-d).

\section{Discussion}

We performed a population-based study of 55 patients with a newly diagnosed glioblastoma, who were treated with current standard treatment. In comparison to the initial registration trial, our patient cohort is a representative cohort with an OS of 14.6 months and a PFS was 10 months [1]. The response to chemoradiation usually tends to follow a normal distribution, while in this patient cohort a distinct subgroup existed that responded longer than 16 months and survived the first 2 years. Similar to other studies, we found younger age, no corticosteroid treatment before start of chemoradiation and low postoperative tumor volume important predictive factors for a favorable treatment response and survival $[3,19]$. In contrast to some other studies, performance status and extent of surgical resection did not predict for treatment outcome.

In general, but for brain tumors in particular, blood represents an attractive platform for prediction and monitoring of treatment response since it is easy to obtain by venous puncture. In previous studies peptide profiles in serum of patients with glioblastoma differed from healthy donors $[10,20]$. Gollapalli and colleagues compared the serum proteome of patients with GBM and healthy subjects and revealed 55 differentially expressed and statistically significant $(\mathrm{p}<0.05)$ protein spots [20]. Several studies demonstrated that serum peptide profiles can be used for diagnostic purposes and early detection of cancer [21, 22]. This indicates that peptide profiles represents a sensitive tool, which may be useful to guide therapeutic decisions in daily practice. Moreover, the serum peptidome was shown to harbor robust biomarkers for prediction of treatment response, including chemoradiation, in other types of cancer [23, 24]. We collected serum from GBM patients postoperative, after chemoradiation, and after completion of the whole treatment. Analysis of the serum peptide profiles did not yield a predictive signature, so we concluded that peptides in serum collected after surgery cannot predict outcome of multimodality treatment. This may be due to the fact that GBM is a heterogeneous disease with different molecular abnormalities that may preclude the ability to identify a single set of proteins that reflects outcome. The total number of peptides identified in this analysis was comparable to the total number of identified peptides in a previous study from our department [14]. They found that serum peptidome profiling may aid in prediction of treatment outcome of patients with advanced non-small cell lung cancer (NSCLC) treated with chemotherapy [14]. Furthermore, the clinical predictive potential of peptide profiling for systemic treatment was shown by Gregorc et al. They found a predictive proteomic signature in patients with NSCLC treated with second-line erlotinib or chemotherapy [26] and supported by multiple studies evaluating MS-based serum and plasma peptidome profiling for prediction of treatment outcome in patients with solid malignancies as summarized previously [27]. Our inability to find a predictive peptide profile for GBM patients may indicate that pre-operative serum might be a more suitable source for this analysis. However, even after optimal resection of a GBM, (microscopic) tumor residue is being left. In addition, patients with advanced NSCLC in the study of Voortman and colleagues were treated with chemotherapy only, and they did not receive any surgery [14]. Others reported that serum peptide profiles can also be used as a sensitive measure for early prediction of disease relapse after surgery $[28,29]$. In this study, we observed no significant distinction between patients with maximal versus subtotal resection in unsupervised cluster analysis [data not shown], suggesting that peptide profiles may have less potential in GBM patients.

Serum YKL-40 concentration has been suggested as a useful biomarker for monitoring disease relapse in GBM patients [30, 31]. YKL-40 was shown to be overexpressed in tumor tissue of patients, enhancing tumor growth and angiogenesis [34]. We found very little variation in YKL-40 protein concentrations in serum of patients throughout the disease course. This is a remarkable finding, since others even reported a transient decrease of protein levels postoperatively, while using the same bioassay [31]. We did not observe any correlation between extent of resection and serum YKL-40 that was previously reported in GBM patients [35]. We also found no correlation at different time points between postoperative tumor volume and serum YKL-40 concentrations.

In contrast to YKL-40, Fetuin-A and haptoglobin are proteins that are also abundantly present in serum of healthy individuals. Fetuin-A was found to be increased, while haptoglobin was demonstrated to be decreased in GBM patients with favorable prognosis $[9,10]$. We observed a similar trend without significance for both molecules in our regression analysis. Significant changes in biomarker concentrations were detected when comparing pre-chemoradiation 
A

Haptoglobin

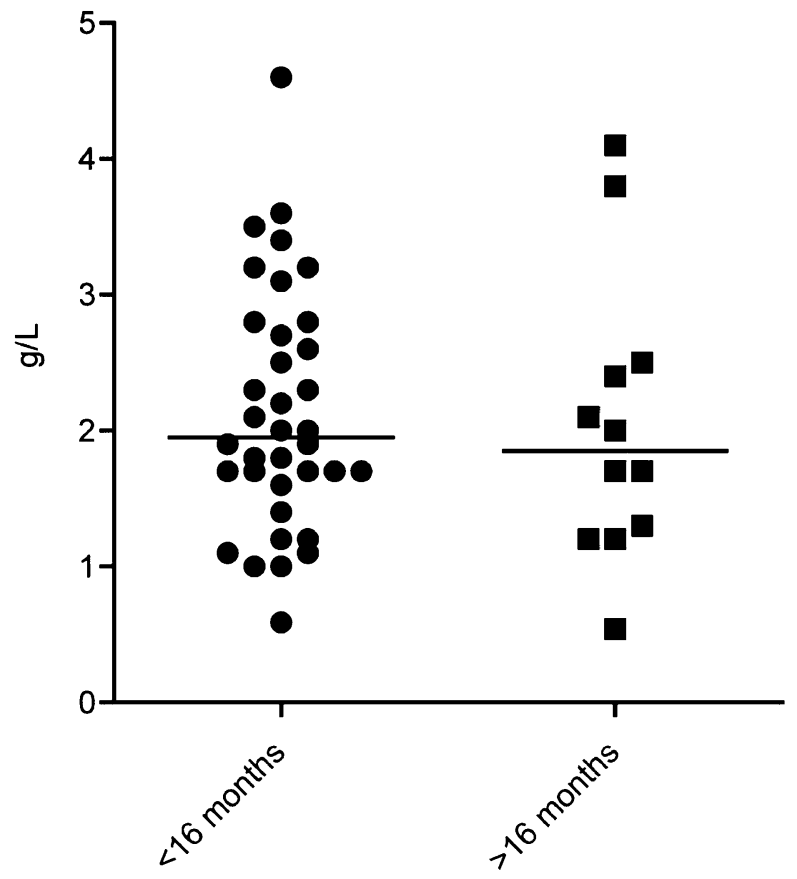

C

Thrombocytes

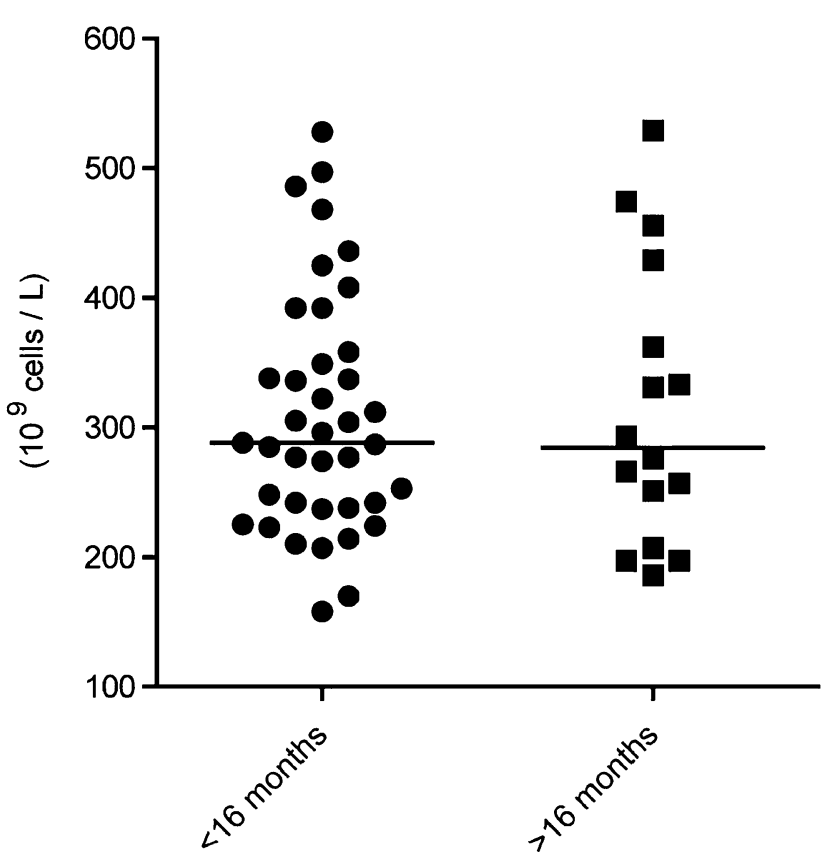

Fig. 4 Analysis of candidate biomarkers and correlation of candidate biomarkers with treatment response. Protein concentrations of previously published candidate biomarkers were measured in serum samples by ELISA (a, d) or immunonefolometry. b Thrombocyte counts were measured by a CD4000 impedance hematology analyzer.

samples with samples taken after completing whole treatment, indicating that timing of sampling may be of importance. Interestingly, Fetuin-A was significantly increased in
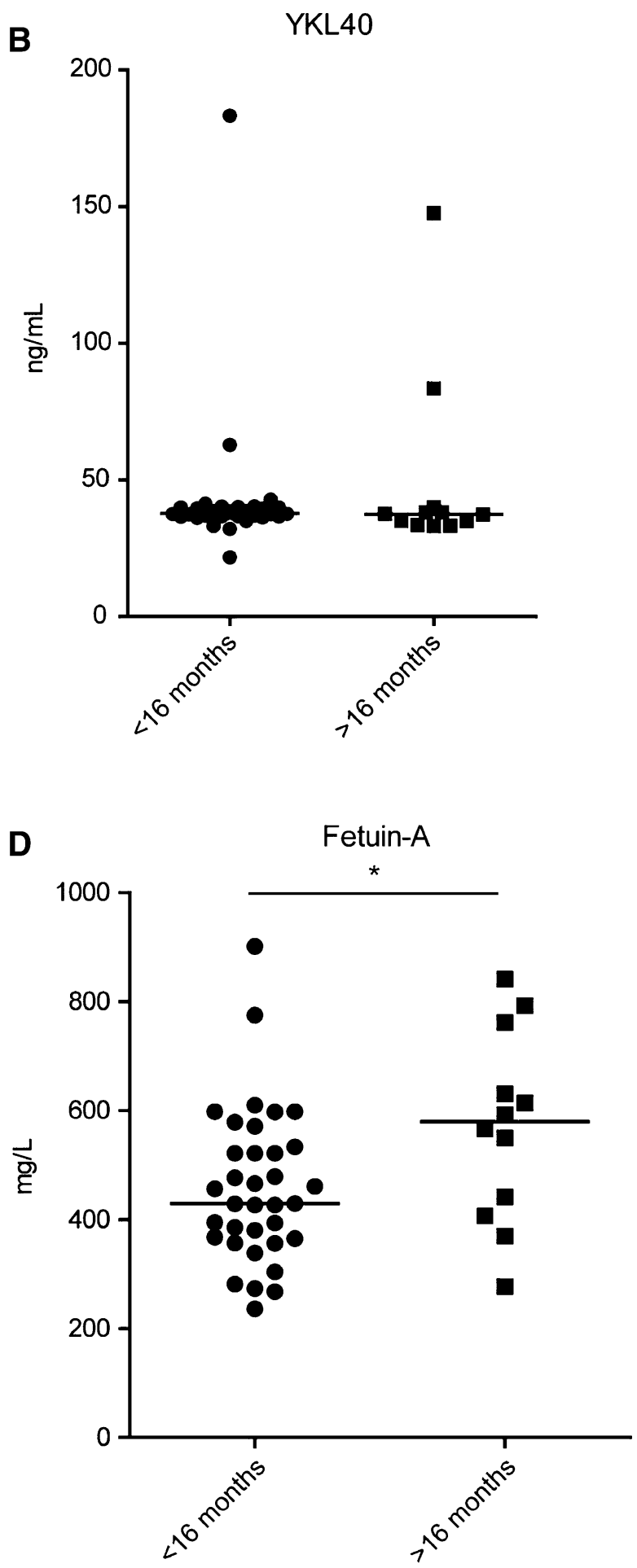

c Correlation with treatment response was determined by Cox regression (not shown) and group comparison. Fetuin-a protein concentrations were significantly different between good and poor response groups $(\mathrm{p}=0.03)$

pre-treatment samples when comparing the poor and good response groups, suggesting that the power of our analysis may have been insufficient. 
Although these biomarkers could still be of value, correlations may be too weak for robust prediction of treatment response. Even, grouping these individual biomarkers in high and low levels did not provide any predictive value (data not shown). All currently established predictive biomarkers are derived from tumor tissue. The advent of large scale genomic profiling allows comprehensive studies of the driver molecules in glioblastoma. For example PDGFR and EGFR were identified as important molecules, together with p53, RB1 and NF1 [36]. Molecular alterations however may change over time, particularly when treatment is involved, urging repeated surgical interventions. The methylation of the MGMT-gene promotor and IDH1 mutations as prognostic and/or predictive factors were not evaluated in our study due to its retrospective nature. Most probably their potential value will not change our inability to find a reliable prognostic or predictive circulating biomarker in serum for patients with GBM.

Still, the non-invasive platforms in blood that are currently in development may become part of future patient management. Circulating tumor cells or extracellular vesicles are shed by tumors into the circulation and may harbor tumor specific molecules [37]. Epigenetic features such as MGMT promoter methylation status are strong predictors of treatment response and may be assessed in serum [38]. In addition, we previously found that platelet contents may provide a biomarker for disease and treatment response evaluations [39]. We have demonstrated that tumor cells transfer (mutant) RNA into blood platelets in vitro and in vivo, and showed that blood platelets isolated from glioma and prostate cancer patients contain the cancer-associated RNA biomarkers EGFRvIII and PCA3, respectively. In addition, gene-expression profiling revealed a distinct RNA signature in platelets from glioma patients compared with normal control subjects [39]. Therefore we are currently sampling platelets from patients with a GBM that undergo first-line treatment. These tools can help monitoring drug resistance, guide therapeutic decisions to improve outcome for patients with GBM.

Open Access This article is distributed under the terms of the Creative Commons Attribution 4.0 International License (http://creativecommons.org/licenses/by/4.0/), which permits unrestricted use, distribution, and reproduction in any medium, provided you give appropriate credit to the original author(s) and the source, provide a link to the Creative Commons license, and indicate if changes were made.

\section{References}

1. Stupp R, Mason WP, van den Bent MJ, Weller M, Fisher B et al (2005) Radiotherapy plus concomitant and adjuvant temozolomide for glioblastoma. N Engl J Med 352(10):987-996

2. Wick W, Platten M, Meisner C, Felsberg J, Tabatabai et al (2012) Temozolomide chemotherapy alone versus radiotherapy alone for malignant astrocytoma in the elderly: the NOA-08 randomised, phase 3 trial. Lancet Oncol 13(7):707-715

3. Weller M, Felsberg J, Hartmann C, Berger H, Steinbach et al (2009) Molecular predictors of progression-free and overall survival in patients with newly diagnosed glioblastoma: a prospective translational study of the German Glioma Network. J Clin Oncol 27(34):5743-5750

4. Petricoin EF, Belluco C, Araujo RP, Liotta LA (2006) The blood peptidome: a higher dimension of information content for cancer biomarker discovery. Nat Rev Cancer 6(12):961-967

5. Villanueva J, Shaffer DR, Philip J, Chaparro CA, ErdjumentBromage $\mathrm{H}$ et al (2006) Differential exoprotease activities confer tumor-specific serum peptidome patterns. J Clin Invest 116(1):271-284

6. Tanwar MK, Gilbert MR, Holland EC (2002) Gene expression microarray analysis reveals YKL-40 to be a potential serum marker for malignant character in human glioma. Cancer Res 62:4364-4368

7. Nutt CL, Betensky RA, Brower MA, Batchelor TT, Louis DN et al (2005) YKL-40 is a differential diagnostic marker for histologic subtypes of high-grade gliomas. Clin Cancer Res 11:2258-2264

8. Nigro JM, Misra A, Zhang L, Smirnov I, Colman H et al (2005) Integrated array-comparative genomic hybridization and expression array profiles identify clinically relevant molecular subtypes of glioblastoma. Cancer Res 65:1678-1686

9. Petrik V, Saadoun S, Loosemore A, Hobbs J, Opstad KS et al (2008) Serum alpha 2-HS glycoprotein predicts survival in patients with glioblastoma. Clin Chem 54(4):713-722

10. Kumar DM, Thota B, Shinde SV, Prasanna KV, Hegde AS et al (2010) Proteomic identification of haptoglobin alpha2 as a glioblastoma serum biomarker: implications in cancer cell migration and tumor growth. J Proteome Res 9(11):5557-5567

11. Williams M, Liu ZW, Woolf D, Hargreaves S, Michalarea et al (2012) Change in platelet levels during radiotherapy with concurrent and adjuvant temozolomide for the treatment of glioblastoma: a novel prognostic factor for survival. J Cancer Res Clin Oncol 138(10):1683-1688

12. Macdonald DR, Cascino TL, Schold SC Jr, Cairncross JG (1990) Response criteria for phase II studies of supratentorial malignant glioma. J Clin Oncol 8(7):1277-1280

13. Jimenez CR, El Filali Z, Knol JC, Hoekman K, Kruyt F et al (2007) Automated serum peptide profiling using novel magnetic C18 beads off-line coupled to MALDI-TOF-MS. ProteomicsClin Appl 1(6):598-604

14. Voortman J, Pham TV, Knol JC, Giaccone G, Jimenez CR (2009) Prediction of outcome of non-small cell lung cancer patients treated with chemotherapy and bortezomib by time-course MALDI-TOF-MS serum peptide profiling. Proteome Sci 7:34

15. Minniti G, Salvati M, Arcella A, Buttarelli F, D’Elia A et al (2011) Correlation between O6-methylguanine-DNA methyltransferase and survival in elderly patients with glioblastoma treated with radiotherapy plus concomitant and adjuvant temozolomide. J Neuro-oncol 102(2):311-316

16. Brandes AA, Franceschi E, Tosoni A, Blatt V, Pession A et al (2008) MGMT promoter methylation status can predict the incidence and outcome of pseudoprogression after concomitant radiochemotherapy in newly diagnosed glioblastoma patients. J Clin Oncol 26(13):2192-2197

17. Wen PY, Macdonald DR, Reardon DA, Cloughesy TF, Sorensen AG et al (2010) Updated response assessment criteria for highgrade gliomas: response assessment in neuro-oncology working group. J Clin Oncol 28(11):1963-1972

18. Gallego Perez-Larraya J, Lahutte M, Petrirena G, Reyes-Botero G, Gonzalez-Aguilar A et al (2012) Response assessment in recurrent glioblastoma treated with irinotecan-bevacizumab: 
comparative analysis of the Macdonald, RECIST, RANO, and RECIST + F criteria. Neuro Oncol 14(5):667-673

19. Gorlia T, van den Bent MJ, Hegi ME, Mirimanoff RO, Weller $M$ et al (2008) Nomograms for predicting survival of patients with newly diagnosed glioblastoma: prognostic factor analysis of EORTC and NCIC trial 26981-22981/CE.3. Lancet Oncol 9(1):29-38

20. Gollapalli K, Ray S, Srivastava R, Renu D, Singh P et al (2012) Investigation of serum proteome alterations in human glioblastoma multiforme. Proteomics 12(14):2378-2390

21. Zhu D, Wang J, Ren L, Li Y, Xu B et al (2013) Serum proteomic profiling for the early diagnosis of colorectal cancer. J Cell Biochem 114(2):448-455

22. Opstal-van Winden AW, Krop EJ, Karedal MH, Gast MC, Lindh $\mathrm{CH}$ et al (2011) Searching for early breast cancer biomarkers by serum protein profiling of pre-diagnostic serum; a nested casecontrol study. BMC Cancer. doi:10.1186/1471-2407-11-381

23. Taguchi F, Solomon B, Gregorc V, Roder H, Gray R et al (2007) Mass spectrometry to classify non-small-cell lung cancer patients for clinical outcome after treatment with epidermal growth factor receptor tyrosine kinase inhibitors: a multicohort cross-institutional study. J Natl Cancer Inst 99(11):838-846

24. Hayashida Y, Honda K, Osaka Y, Hara T, Umaki T et al (2005) Possible prediction of chemoradiosensitivity of esophageal cancer by serum protein profiling. Clin Cancer Res 11(22):8042-8047

25. Liao CC, Mehta A, Ward NJ, Marsh S, Arulampalam T et al (2010) Analysis of post-operative changes in serum protein expression profiles from colorectal cancer patients by MALDITOF mass spectrometry: a pilot methodological study. World J Surg Oncol. doi:10.1186/1477-7819-8-33

26. Gregorc V, Novello S, Lazzari C, Barni S, Aieta M et al (2014) Predictive value of a proteomic signature in patients with nonsmall-cell lung cancer treated with second-line erlotinib or chemotherapy (PROSE): a biomarker-stratified, randomised phase 3 trial. Lancet Oncol 15(7):713-721

27. Labots M, Schütte LM, van der Mijn JC, Pham TV, Jiménez CR et al (2014) Mass spectrometry-based serum and plasma peptidome profiling for prediction of treatment outcome in patients with solid malignancies. Oncologist 19(10):1028-1039
28. Mordhorst LB, Sorbe B, Ahlin C (2012) A study of serum biomarkers associated with relapse of cervical cancer. Anticancer Res 32(11):4913-4922

29. Gourin CG, Zhi W, Adam BL (2009) Proteomic identification of serum biomarkers for head and neck cancer surveillance. Laryngoscope 119(7):1291-1302

30. Iwamoto FM, Hottinger AF, Karimi S, Riedel E, Dantis J et al (2011) Serum YKL-40 is a marker of prognosis and disease status in high-grade gliomas. Neuro Oncol 13(11):1244-1251

31. Hormigo A, Gu B, Karimi S, Riedel E, Panageas KS et al (2006) YKL-40 and matrix metalloproteinase- 9 as potential serum biomarkers for patients with high-grade gliomas. Clin Cancer Res 12(19):5698-5704

32. Chupp GL, Lee CG, Jarjour N, Shim YM, Holm CT et al (2007) A chitinase-like protein in the lung and circulation of patients with severe asthma. N Engl J Med 357(20):2016-2027

33. Kastrup J, Johansen JS, Winkel P, Hansen JF, Hildebrandt P et al (2009) High serum YKL-40 concentration is associated with cardiovascular and all-cause mortality in patients with stable coronary artery disease. Eur Heart J 30(9):1066-1072

34. Francescone RA, Scully S, Faibish M, Taylor SL, Oh D et al (2011) Role of YKL-40 in the angiogenesis, radioresistance, and progression of glioblastoma. J Biol Chem 286(17):15332-15343

35. Bernardi D, Padoan A, Ballin A, Sartori M, Manara R et al (2012) Serum YKL-40 following resection for cerebral glioblastoma. J Neurooncol 107(2):299-305

36. Cancer Genome Atlas Research Network (2008) Comprehensive genomic characterization defines human glioblastoma genes and core pathways. Nature 455(7216):1061-1068

37. Shao H, Chung J, Balaj L, Charest A, Bigner DD et al (2012) Protein typing of circulating microvesicles allows real-time monitoring of glioblastoma therapy. Nat Med 18(12):1835-1840

38. Liu BL, Cheng JX, Zhang W, Zhang X, Wang R et al (2010) Quantitative detection of multiple gene promoter hypermethylation in tumor tissue, serum, and cerebrospinal fluid predicts prognosis of malignant gliomas. Neuro Oncol 12(6):540-548

39. Nilsson RJ, Balaj L, Hulleman E, van Rijn S, Pegtel DM et al (2011) Blood platelets contain tumor-derived RNA biomarkers. Blood 118(13):3680-3683 